\title{
A New Fuzzy Skeletonization Algorithm and Its Applications to Medical Imaging
}

\author{
Dakai Jin $^{1}$ and Punam K. Saha ${ }^{1,2}$ \\ ${ }^{1}$ Department of Electrical and Computer Engineering, University of Iowa, USA \\ ${ }^{2}$ Department of Radiology, University of Iowa, USA \\ \{dakai-jin, punam-saha\} @uiowa.edu
}

\begin{abstract}
Skeletonization provides a simple yet compact representation of an object and is widely used in medical imaging applications including volumetric, structural, and topological analyses, object representation, stenoses detection, path-finding etc. Literature of three-dimensional skeletonization is quite matured for binary digital objects. However, the challenges of skeletonization for fuzzy objects are mostly unanswered. Here, a framework and an algorithm for fuzzy surface skeletonization are developed using a notion of fuzzy grassfire propagation which will minimize binarization related data loss. Several concepts including fuzzy axial voxels, local and global significance factors are introduced. A skeletal noise pruning algorithm using global significance factors as significance measures of individual branches is developed. Results of application of the algorithm on several medical objects have been illustrated. A quantitative comparison with an ideal skeleton has demonstrated that the algorithm can achieve sub-voxel accuracies at various levels of noise and downsampling. The role of fuzzy skeletonization in thickness computation at relatively low resolution has been demonstrated.
\end{abstract}

\section{Introducation}

Availability of a wide spectrum of medical imaging techniques together with routine production of large image datasets for both clinical and research purposes have intensified the image processing needs for computerized extraction of knowledge from acquired images. A common objective of medical imaging is to extract information of internal human organ or tissue through in vivo or ex vivo imaging. Often, these images are processed through complex cascades of processing and analysis steps. Skeletonization is a transformation process that reduces a volumetric object into a significantly reduced, simplified and compact representation, referred to as "medial axis" or "skeleton", while preserving the topology [1] and geometric properties of the object.

Skeletonization has been widely used in different medical imaging applications, including, thickness computation [2], topological classification [3,4], path finding [5], and object shape modeling [6]. Many 3D skeletonization algorithms [7-11] have been reported for binary digital objects. But the same is not true for fuzzy skeletonization. Although, a few works on gray scale skeletonization have been presented in literature [12-15], the fundamental challenges related to fuzzy skeletonization in the presence of 
partial voluming and degradation of object are mostly unanswered and a complete skeletonization algorithm for fuzzy digital objects is missing. For example, the fuzzy skeletonization algorithm by Pal [15], first, binarized a fuzzy object under optimization of fuzzy compactness [14] and then a binary skeletonization algorithm is applied. Yim et al. [12] used a significant path selection approach in an ordered region growing (ORG) graph; although, this approach does not use binarization, it is not obvious how to generalize this idea to extract the skeleton of a surface-like object. Sanniti di Baja et al. [13] used 3D convertion of a 2D grey image by uplifting each 2D voxel by a height equivalent to its grey-value and then followed a 3D binary skeletonization and collapsing from 3D to 2D. Here, a framework and an algorithm for fuzzy surface skeletonization are developed using a new notion of fuzzy grassfire propagation. The process of fuzzy grassfire propagation is simulated using fuzzy distance transform (FDT) [16]. Arcelli and Sanniti di Baja [17] first used DT in skeletonization in 2-D and discussed its advantages. Saito and Torowaki [9] and others [10] have used DT to define the voxel erosion sequence. Here, FDT is used to define the fuzzy grassfire propagation. Several new concepts including fuzzy axial voxels, local and global significance factors are introduced in this paper. Also, a skeletal noise pruning algorithm using significance measures at individual branch level is developed and its effectiveness is experimentally demonstrated.

\section{Fuzzy Skeletonization Theory and Algorithm}

Blum's pioneering work on grassfire transform [18] led to the notion of skeletonization that converts a volumetric object into a union of surfaces and curves. The process is defined using fire propagation on a grass field, where the field resembles an object. The fire is simultaneously set at all boundary points and it propagates inwardly at a uniform speed. The skeleton is defined as the set of quench points where two or more fire fronts meet. However, the notion of skeletonization for fuzzy objects has not yet been defined. To define a fuzzy skeletonization process, we suggest modifying the Blum's grassfire transform for a fuzzy object where the membership function is interpreted as local material density so that the speed of grassfire at a given point is inversely proportional to its material density. Following this notion, it can be shown that fuzzy distance transform (FDT) [16] value at a point $p$ is proportional to the time when the fire front reaches $p$. Therefore, during the fuzzy grassfire propagation, the speed of a fire front at a point equates to the inverse of local material density and this equality is violated only at quench points where the propagation process is interrupted. Thus, a voxel $p \in Z^{3}$, where $Z$ is the set of integers and $Z^{3}$ represents a rectangular image grid, is a fuzzy quench voxel in a fuzzy digital object $\mathcal{O}=$ $\left\{\left(p, f_{\mathcal{O}}(p)\right) \mid p \in Z^{3} \wedge f_{\mathcal{O}}: Z^{3} \rightarrow[0,1]\right\}$ if the following inequality holds for every neighbor $q$ of $p$

$$
F D T(q)-F D T(p)<\frac{1}{2}\left(f_{\mathcal{O}}(p)+f_{\mathcal{O}}(q)\right)|p-q| .
$$

Saha and Wehrli [19] introduced the above definition of fuzzy quench voxel which was further studied by Svensson [20] where she referred to it as the center of fuzzy maximal ball (CFMB). Also, it may be noted that the definition of fuzzy quench voxel is equivalent to that of center of maximal ball (CMB) [21] for binary digital objects. 


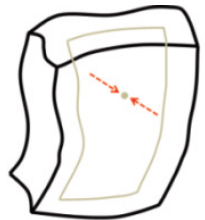

(a)

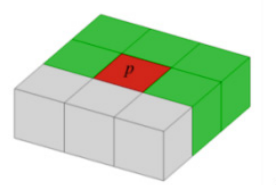

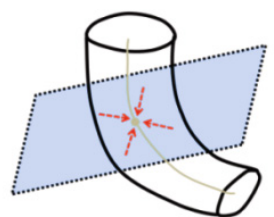

(b)

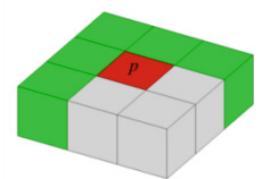

(c)

Fig. 1. (a,b) Illustration of independent fire fronts meeting at surface-like (a) and curve-like (b) quench points. (c) Two example masks used to compute the significance of a surface quench voxel. Voxels colored in green are used for average significance computation. Four coplanar geometrically identical masks may be constructed from each of these two examples.

During the process of fuzzy skeletonization, voxels are removed in the increasing order of their FDT values. The overall fuzzy skeletonization process is summarized in the following. Here, $O=\left\{p \mid f_{\mathcal{O}}(p)>0\right\}$ denotes the support of the fuzzy object $\mathcal{O}$.

\section{Primary skeletonization}

select voxels $p \in O$ in the order of FDT values

if $p$ is not a fuzzy axial voxel and its deletion preserves 3D topology and 2D topology on mid-planes remove $p$ from $O$, i.e., set $f_{\mathcal{O}}(p)=0$

\section{Final skeletonization:}

select voxels $p \in O$ in the order of FDT values

if $p$ is in two-voxel thick structure and its deletion

preserves 3D topology and 2D topology on mid-planes

remove $p$ from $O$, i.e., set $f_{\mathcal{O}}(p)=0$

select voxels $p \in O$ in the order of FDT values

if topologic and geometric features of $p$ fail to agree

and its deletion preserves 3D topology

remove $p$ from $O$, i.e., set $f_{\mathcal{O}}(p)=0$

This notion of primary and final skeletonization was simultaneously introduced by Saha et al. [8] and Arcelli et al. [21]. Saha et al. described it in 3-D while Arcelli et al. presented the idea in 2-D for a DT-based skeletonization algorithm.

Removal of a voxel $p \in O$ preserves the topology of $O$ if and only if $p$ is a $(26,6)$ simple voxel [1] in $O$. Beside the 3D topology preservation condition, an additional constrain of 2D topology preservation in all three middle planes of the candidate voxel is subjected to ensure continuity of surface-like structures and to avoid undesired drilling effects as illustrated by Saha et al. [8].

In the following, several new concepts including fuzzy axial voxels, local and global significance factors and two-voxel thick structures are introduced for fuzzy 
objects. Also, a condition defining the disagreement between topological and geometric features of a voxel is provided. Finally, a new noisy skeletal branches pruning algorithm based on global significance factor (GSF) is presented.

\subsection{Fuzzy Axial Voxel}

Two types of quench points may form -surface- and curve-quench points (Fig. $1(a, b))$. A surface quench point is formed when two opposite fire fronts meet while a curve quench point is formed when fire fronts meet from all directions on a plane. In a digital space, surface-quench voxel is formed when two opposite fire fronts meet along $\mathrm{x}$-, $\mathrm{y}$ - or $\mathrm{z}$-direction and a curve-quench voxel is formed when fire fronts meet from all eight directions in xy-, yz-, or zx-planes. A voxel $p=\left(p_{x}, p_{y}, p_{z}\right) \in O$ is an $x$-surface-quench voxel if the following two conditions are satisfied for $p_{x_{-}}=$ $\left(p_{x}-1, p_{y}, p_{z}\right), p_{x+}=\left(p_{x}+1, p_{y}, p_{z}\right)$, and $p_{x++}=\left(p_{x}+2, p_{y}, p_{z}\right)$ :

1) $\operatorname{FDT}(p)>\operatorname{FDT}\left(p_{x_{-}}\right)$,

2) $\operatorname{FDT}(p)>\operatorname{FDT}\left(p_{x+}\right) \vee\left(F D T(p)=F D T\left(p_{x+}\right) \wedge F D T(p)>F D T\left(p_{x++}\right)\right)$.

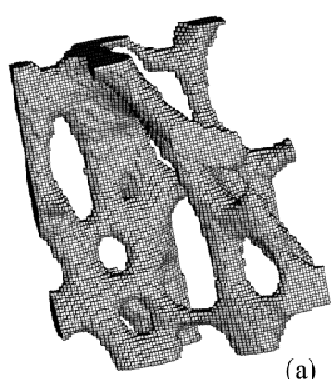

(a)

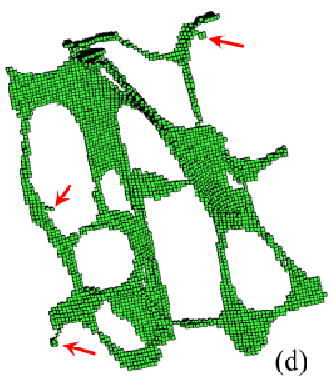

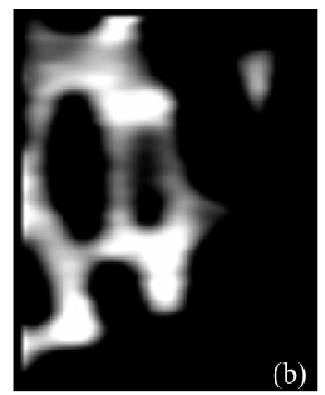

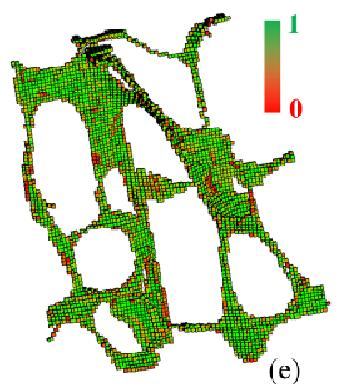

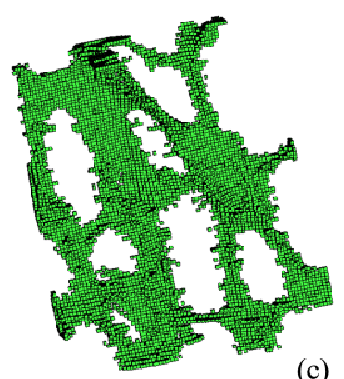

(c)

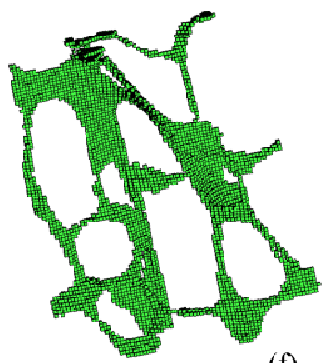

(f)

Fig. 2. Results of intermediate steps of fuzzy skeletonization. (a) 3D display of trabecular bone region in a micro-CT image of a cadaveric distal tibia specimen. (b) A sagittal image slice displaying the fuzziness in the image. (c) All quench voxels before filtering with local significance measure. (d) Results of final skeleton; noisy branches are indicated by red arrows. (e) Results of local significance computation. (g) Final results after noisy branch pruning.

To define a curve-quench voxel, let us first consider the formulation of the situation when fire fronts meet in the xy-plane. Curve quench voxels may form a $2 \times 2$ 
clique on the xy-plane.Let $P_{\mathrm{xy}}=\left\{\left(p_{x}, p_{y}, p_{z}\right),\left(p_{x}+1, p_{y}, p_{z}\right),\left(p_{x}, p_{y}+1, p_{z}\right),\left(p_{x}+\right.\right.$ $\left.\left.1, p_{y}+1, p_{z}\right)\right\}$ denote the $2 \times 2$ clique. Let $Q\left(P_{\mathrm{xy}}\right)$ denote the set of voxels within the $2 \mathrm{x} 2$ clique $P_{\mathrm{xy}}$ with their FDT value identical to that of $p$, i.e.,

$$
Q\left(P_{x y}\right)=\left\{q \mid q \in P_{x y} \wedge F D T(q)=F D T(p)\right\} .
$$

Thus, the fire front reaches simultaneously at every voxel of $Q\left(P_{\mathrm{xy}}\right)$ from all directions on the xy-plane. Therefore, a voxel $p=\left(p_{x}, p_{y}, p_{z}\right) \in O$ is an $x y$-curvequench voxel if the following condition holds for $\forall q \in M_{\mathrm{xy}}(p)-Q\left(P_{\mathrm{xy}}\right)$

$$
q \text { is 26-adjacent to } Q\left(P_{\mathrm{xy}}\right) \text { implies } F D T(q)<F D T(p),
$$

where, $M_{\mathrm{xy}}(p)$ is the set of all voxels constructing the xy-plane through $p$.

Although the quench voxels captures the notion of fuzzy grassfire transform, it suffers from the fact that a large number of spurious quench voxels are created (Fig. 2(c)). Therefore, it is imperative to filter out some of these quench voxels based on their significance. Here, we introduce a function that resembles the "local significance factor" (LSF) of individual voxels and use LSF measures in the neighborhood to determine the significance of a quench voxel. Local significance factor or LSF of any voxel $p \in O$, denoted by $\operatorname{LSF}(p)$, is defined as follows:

$$
\operatorname{LSF}(p)=1-f_{+}\left(\max _{q \in N^{*}(p)} \frac{F D T(q)-F D T(p)}{\frac{1}{2}\left(f_{\mathcal{O}}(p)+f_{\mathcal{O}}(q)\right)|p-q|}\right),
$$

where the function $f_{+}(x)$ returns the value of $x$ if $x>0$ and zero otherwise, and $N^{*}(p)$ is the set of neighbors of $p$. The term inside the function $f_{+}$essentially represents the inverse of speed of fire front propagation at the voxel $p$ normalized by local material density. The above formulation of LSF is used to determine the significance of a quench voxel. Let $p=\left(p_{x}, p_{y}, p_{z}\right)$ be an x-surface quench voxel. To compute the support for $p$, first, a projection of three voxels $\left\{q_{i, j}^{+}=\left(p_{x}-1, p_{y}+\right.\right.$ $\left.\left.i, p_{z}+j\right), q_{i, j}=\left(p_{x}, p_{y}+i, p_{z}+j\right), q_{i, j}^{-}=\left(p_{x}+1, p_{y}+i, p_{z}+j\right)\right\} \quad, \quad$ for $\quad$ some $i, j \in\{-1,0,1\}$, is computed to generate a $3 \times 3$ field of significance map $M_{p}^{\mathrm{x}}(i, j)$ as follows:

$$
M_{p}^{\mathrm{x}}(i, j)=\max \left\{\operatorname{LSF}\left(q_{i, j}^{+}\right), \operatorname{LSF}\left(q_{i, j}\right), \operatorname{LSF}\left(q_{i, j}^{-}\right)\right\} .
$$

To determine the significance of an $\mathrm{x}$-surface quench voxel $p$, the average significance value $m_{i}^{\mathrm{x}}$ over each of eight different masks $D_{i} \mid i=1, \cdots, 8$ (see Fig. 1(c)) is computed. An x-surface-quench voxel $p$ is referred to as $x$-significant surfacequench voxel, if any of the average values $m_{i}^{\mathrm{x}} \mid i=1, \cdots, 8$ is greater than a preset threshold. It may be noted that each of the mask $D_{i}$ contains five ' 1 ' values (green) which are used for computation of average significance $m_{i}^{\mathrm{x}}$ and all green voxels in the mask fall on one side of $p$. A voxel $p \in A$ is referred to as a significant surfacequench voxel if it is an $\mathrm{x}-, \mathrm{y}-$, or $\mathrm{z}$-significant surface-quench voxel.

Significance of an xy-curve-quench voxel $p=\left(p_{x}, p_{y}, p_{z}\right)$ is defined by the maximum LSF value on either of the two $3 \mathrm{x} 3$ planar cliques $C_{\mathrm{z}}^{+}=\left\{\left(p_{x}+i, p_{y}+\right.\right.$ $\left.\left.j, p_{z}+1\right) \mid i, j \in\{-1,0,1\}\right\} \quad$ and $\quad C_{\mathrm{z}}^{-}=\left\{\left(p_{x}+i, p_{y}+j, p_{z}-1\right) \mid i, j \in\{-1,0,1\}\right\}$. 
Specifically, $p$ is an xy-significant curve-quench voxel if the largest LSF value in either of the two cliques $\mathrm{C}_{\mathrm{z}}^{+}$and $\mathrm{C}_{\mathrm{z}}^{-}$is greater than a preset threshold. An xy-, yz-, or zX-significant curve-quench voxel is referred to as a significant curve-quench voxel. A significant surface- or curve-quench voxel is referred to as a fuzzy axial voxel.

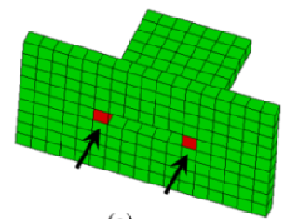

(a)

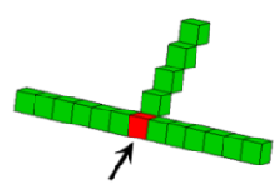

(b)

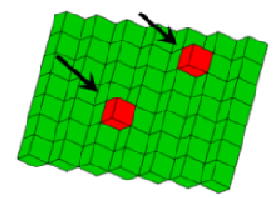

(c)

Fig. 3. Examples of voxels with contradiction between their topological and geometric properties. Red voxels are simple voxels which may appear at surface edge (a) or at curve end (b,c). However, these voxels fail to satisfy the geometric properties of a surface edge or a curve end.

\subsection{Final Skeletonization}

Final skeletonization converts two-voxel thick structures into one-voxel thick structures and it is completed in two steps. Intuitively, a voxel is two-voxel thick if its three non-opposite 6-neighbors are skeletal voxels and forms two-voxel thick surface across $\mathrm{x}-$, $\mathrm{y}$-, and/or z-direction [8]. During the first step, thick voxels are considered for erosion in the order of their FDT values. Specifically, a voxel satisfying two-voxel thickness along all three coordinate directions is deleted if it is a $(26,6)$ simple voxel. A voxel satisfying two-voxel thickness along two directions, say $\mathrm{x}$ and $\mathrm{y}$, is deleted if it is a $(26,6)$ simple voxel and it preserves $2 \mathrm{D}$ topology in $M_{\mathrm{xy}}(p)$. Finally, a voxel satisfying two-voxel thickness along only one directions, say $\mathrm{x}$, is deleted if it is a $(26,6)$ simple voxel and it preserves $2 \mathrm{D}$ topology in both $M_{\mathrm{xy}}(p)$ and $M_{\mathrm{zx}}(p)$.

The second step of final skeletonization brings a new idea of removing voxels with contradicting topological and geometric properties. Specifically, simple voxels must appears at a surface edge or at a curve end. Therefore, a simple voxel is deleted if it fails to satisfy geometric properties of a surface edge or a curve end. For example, for Fig. 3(a), the red voxels do not have two opposite 6-neighbors in background to satisfy the geometry of a digital surface. Similarly, for Fig. 3(b,c), the singleton simple voxels (red) are expected to appear at curve end; but, there is no plane where are isolated failing to satisfy the geometric property of a curve end.

\subsection{Skeleton Pruning}

The goal of a skeleton pruning algorithm is to discriminate between significant and non-significant branches so that only false branches may be removed. This goal is accomplished by computing LSF-weighted length of an individual branch from its edge to the corresponding junction voxel. This LSF-weighted branch length is used as a global significance factor (GSF) of a specific skeletal branch. This overall process is implemented using the following steps - (1) digital topological analysis (DTA), (2) conversion of two-voxel wide curve-like structures into a true digital curve, (3) computation of GSF for all branches, and (4) removal of non-significant branches. 


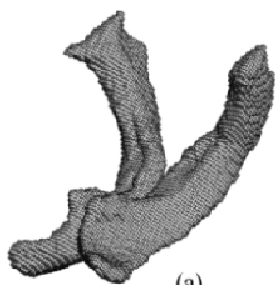

(a)

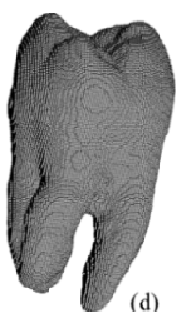

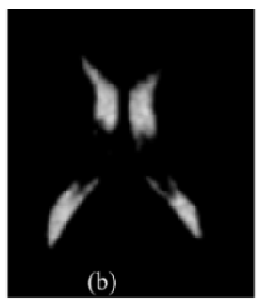

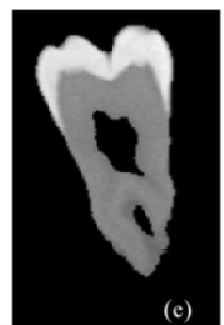

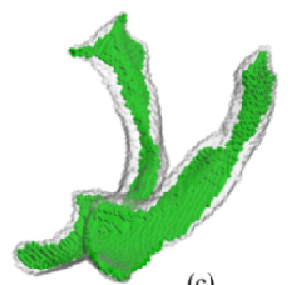

(c)

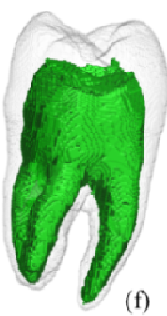

Fig. 4. Results of application of fuzzy skeletonization on two anatomic structures fuzzy segmented from acquired images. (a) A part of cerebrospinal fluid segmented from human brain MR imaging. (b) An axial image slice illustrating the fuzziness and noise. (c) Surface rendering of the fuzzy skeleton. (d-f) Same as (a-c) but for micro CT data of a human tooth.

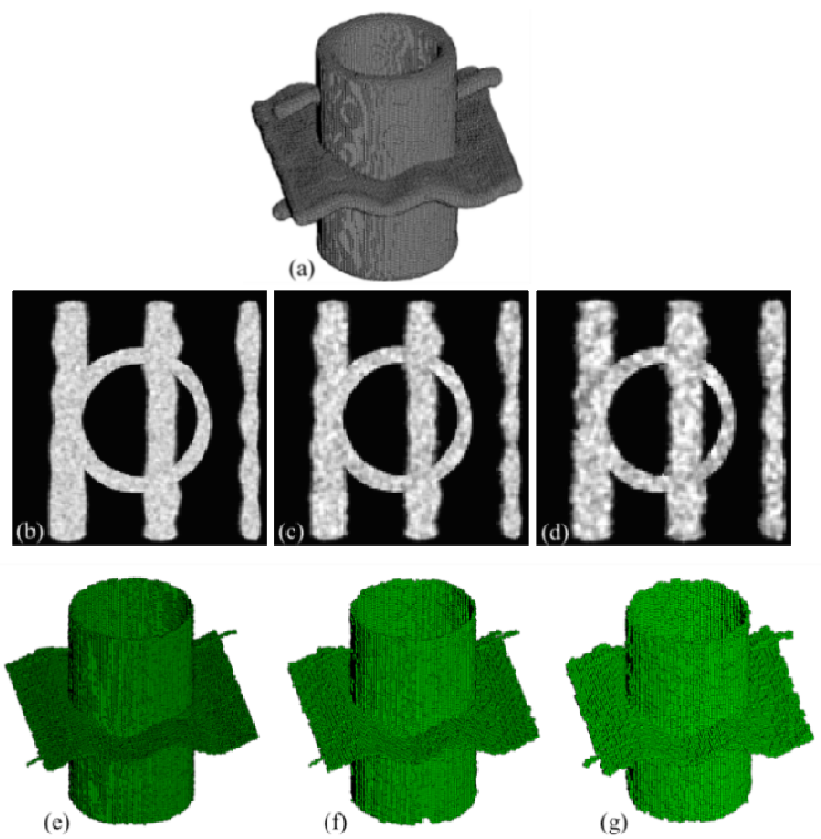

Fig. 5. Results of application of fuzzy skeletonization on a phantom data at different levels of noise and down sampling. (a) 2D display of the original phantom. (b-d) Axial image slices at SNR of 24, 12 and 6 and downsampling of three, four, and five voxels. (e-g) Results of fuzzy skeletonization of the phantom at three levels of noise and downsampling of (b-d). 


\section{Experiment Results}

Results of intermediate steps of steps of skeletonization and pruning on a small region of trabecular bone image are illustrated in Fig. 2. Results of application of skeletonization and pruning of two other medical images are presented in Fig. 4. For all these examples, the results of skeletonization and pruning are visually encouraging.

To quantitatively examine the accuracy of the method, we generated a 3D binary object and its ground truth skeleton at high resolution. Then test phantom images were generated from the high resolution binary image at different downsampling and signal-to-noise ratio (SNR). Error was calculated by comparing computed and true skeletons. The high resolution image and true skeleton was generated by sampling an ideal skeleton in the continuous 3-D space $R^{3}$. Let $S_{\mathrm{T}}$ be the set of $N_{\mathrm{T}}$ number of sampled points; $S_{\mathrm{T}}$ is considered as the true skeleton. A Euclidean distance transform $D T_{\mathrm{T}}: Z^{3} \rightarrow R^{+}$is computed from $S_{\mathrm{T}}$. To generate a binary object with non-uniform thickness value, a smooth thickness field $f_{\text {thickness }}: Z^{3} \rightarrow R^{+}$is computed. Finally, the volumetric object is defined as the set of all voxels with its $D T_{\mathrm{T}}$ value less than or equal to the local thickness value $f_{\text {thickness }}$. Three high resolution binary objects with true skeletons were generated and used for this experiment. Their original image was generated in a 500×500×500 array. An example binary object is shown in Fig. 5. Let $S_{C}^{l, \rho}$ denote the computed skeleton at the downsampling rate of $l$ and the noise at SNR of $\rho$ and let $N_{C}^{l, \rho}$ denote the number of voxels in $S_{C}^{l, \rho}$. The skeletonization error is computed by the following equation:

$$
\operatorname{Error}_{l, \rho}=\frac{1}{2 N_{\mathrm{T}}} \sum_{p \in S_{\mathrm{T}}} \min _{q \in S_{C}^{l, \rho}}|p-q|+\frac{1}{2 N_{C}^{l, \rho}} \sum_{p \in S_{C}^{l, \rho}} \min _{q \in S_{\mathrm{T}}}|p-q| .
$$

The average error for phantoms at each level of noise and downsampling is presented in Table 1. As shown in the table, the average error is less than a voxel and as shown by Saha et al. [2], the error of digitization is close to 0.38 voxel. Therefore, after deducting the digitization error, the performance of the fuzzy skeletonization algorithm even at the highest level of noise and downsampling is encouraging.

Table 1. Skeletonization errors at different levels of noise and downsampling

\begin{tabular}{c|llll}
$\begin{array}{c}\text { Different } \\
\text { downsampling }\end{array}$ & noise free & Different signal to noise ratio \\
\hline $3 \times 3 \times 3$ & 0.486 & 0.516 & 0.540 & 0.576 \\
$4 \times 4 \times 4$ & 0.520 & 0.534 & 0.545 & 0.578 \\
$5 \times 5 \times 5$ & 0.573 & 0.577 & 0.587 & 0.595 \\
\hline
\end{tabular}

Finally, the application of fuzzy skeletonization in thickness computation is demonstrated in Fig. 6. Here, the basic principle of FDT-based thickness computation by Saha and Wehrli [2] is adopted except the fact that fuzzy skeletonization is used instead of binary skeletonization. Here, FDT-based depth values are sampled along the skeleton of the target object; thus providing the regional thickness distribution over the object. Let $S_{\text {skeleton }}$ denote the set voxels in the fuzzy skeleton of a fuzzy object 
$\mathcal{O}$ and let FDT give the fuzzy distance transform map. At each skeletal voxel $p \in S_{\text {skeleton, }}$ its thickness value $\tau(p)$ is computed as twice the FDT value $F D T(p)$. Finally, thickness values at non-skeletal voxels are inherited from the nearest skeletal voxel using the feature propagation algorithm introduced in [4]. Results of thickness distribution shown in Fig. 6 are visually satisfactory.

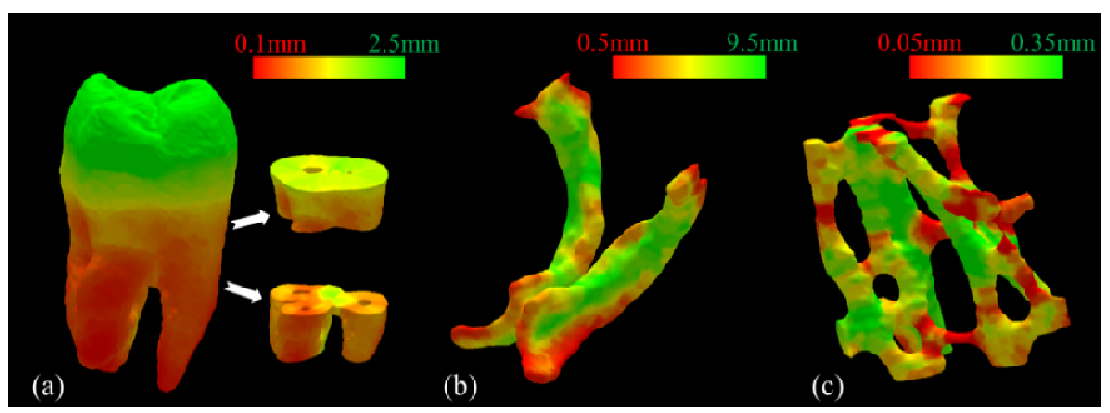

Fig. 6. Results of local thickness computation for three anatomic objects using fuzzy skeletonization, FDT, and feature propagation as described in the text

\section{Conclusion}

In this paper, a theoretical framework and a robust algorithm for fuzzy surface skeletonization are developed using a new notion of fuzzy grassfire propagation. The process of fuzzy grassfire propagation has been defined and the equation for fuzzy quench voxels has been formulated. Several new concepts including fuzzy axial voxels, local and global significance factors related to fuzzy skeletonization have been introduced and their theoretical formulations have been presented. A skeletal noise pruning algorithm using significance measures of individual branches has been developed and its performance has been demonstrated. Results of application of the algorithm on several medical objects have been illustrated. A quantitative comparison with an ideal skeleton has demonstrated that the algorithm can achieve sub-voxel accuracies at various levels of noise and downsampling rate. The application of fuzzy skeletonization in thickness computation at relatively low resolution has been demonstrated and the results are visually satisfactory.

Acknowledgements. This work was supported by the NIH grant R01-AR054439.

\section{References}

1. Saha, P.K., Chaudhuri, B.B.: Detection of 3-D simple points for topology preserving transformation with application to thinning. IEEE Trans.on PAMI 16, 1028-1032 (1994)

2. Saha, P.K., Wehrli, F.W.: Measurement of trabecular bone thickness in the limited resolution regime of in vivo MRI by fuzzy distance transform. IEEE Trans. on MI 23, 53-62 (2004) 
3. Saha, P.K., Gomberg, B.R., Wehrli, F.W.: Three-dimensional digital topological characterization of cancellous bone architecture. IJIST 11, 81-90 (2000)

4. Saha, P.K., Xu, Y., Duan, H., Heiner, A., Liang, G.: Volumetric topological analysis: a novel approach for trabecular bone classification on the continuum between plates and rods. IEEE Trans. on MI 29, 1821-1838 (2010)

5. Wan, M., Liang, Z., Ke, Q., Hong, L., Bitter, I., Kaufman, A.: Automatic centerline extraction for virtual colonoscopy. IEEE Trans. on MI 21, 1450-1460 (2002)

6. Pizer, S.M., Fritsch, D.S., Yushkevich, P.A., Johnson, V.E., Chaney, E.L.: Segmentation, registration, and measurement of shape variation via image object shape. IEEE Trans. Med. Imaging 18, 851-865 (1999)

7. Ma, C.M., Sonka, M.: A fully parallel 3D thinning algorithm and Its applications. Computer Vision Image Understanding 64, 420-433 (1996)

8. Saha, P.K., Chaudhuri, B.B., Dutta Majumder, D.: A new shape preserving parallel thinning algorithm for 3D digital images. Pattern Recognition 30, 1939-1955 (1997)

9. Saito, T., Toriwaki, J.-I.: A sequential thinning algorithm for three dimensional digital pictures using the Euclidean distance transformation. In: Proc. 9th SCIA, Uppsala, Sweden, pp. 507-516 (1995)

10. Arcelli, C., Sanniti di Baja, G., Serino, L.: Distance-driven skeletonization in voxel images. IEEE Trans. on PAMI 33, 709-720 (2011)

11. Tsao, Y.F., Fu, K.S.: A parallel thinning algorithm for 3D pictures. Computer Graphics and Image Processing 17, 315-331 (1981)

12. Yim, P.J., Choyke, P.L., Summers, R.M.: Gray-scale skeletonization of small vessels in magnetic resonance angiography. IEEE Trans. on MI 19, 568-576 (2000)

13. di Baja, G.S., Nyström, I., Borgefors, G.: Discrete 3D tools applied to 2D grey-level images. In: Roli, F., Vitulano, S. (eds.) ICIAP 2005. LNCS, vol. 3617, pp. 229-236. Springer, Heidelberg (2005)

14. Pal, S.K., Rosenfeld, A.: Image enhancement and thresholding by optimization of fuzzy compactness. Pat. Rec. Let. 7, 77-86 (1988)

15. Pal, S.K.: Fuzzy skeletonization of an image. Pat. Rec. Let. 10, 17-23 (1989)

16. Saha, P.K., Wehrli, F.W., Gomberg, B.R.: Fuzzy distance transform: theory, algorithms, and applications. Computer Vision and Image Understanding 86, 171-190 (2002)

17. Arcelli, C., di Baja, G.S.: A width-independent fast thinning algorithm. IEEE Trans. on PAMI 7, 463-474 (1985)

18. Blum, H.: A transformation for extracting new descriptors of shape (Models for the Perception of Speech and Visual Form). MIT Press, Cambridge (1967)

19. Saha, P.K., Wehrli, F.W.: Fuzzy distance transform in general digital grids and its applications. In: Proc. 7th JCIS, Research Triangular Park, NC (2003)

20. Svensson, S.: Aspects on the reverse fuzzy distance transform. Pat. Rec. Let. 29, 888-896 (2008)

21. Arcelli, C., di Baja, G.S.: Well-shaped, stable, and reversible skeletons from the $(3,4)-$ distance transform. Journal of Visual Communication and Image Representa 